January 20, 2011

\title{
Rationale Erklärungen für Immobilienpreis-Bubbles: Die Auswirkungen von Risikoschocks auf die Wohnimmobilienpreisvolatilität und die Volatilität von Investitionen in Wohnimmobilien*
}

\begin{abstract}
Die enormen Schwankungen der weltweiten Wohnimmobilienmärkte in den vergangenen Jahren werden in den Medien häufig als Bubbles beschrieben, die hauptsächlich durch irrational exuberance verursacht wurden und deren Ursache in den Liberalisierungstendenzen bei der Finanzierung von Wohnimmobilien zu suchen ist (z. B. Abbau von Finanzmarktregulierungen in den USA, Subprime Hypotheken und strukturierte Finanzmarktprodukte). Entsprechend der Ergebnisse von Dorofenko et al (2011) zeigt diese Arbeit jedoch, dass viele Charakteristika des Wohnimmobilienmarktes, speziell die Volatilitäten der U.S. Wohnimmobilienpreise und die Volatilitäten der Investitionen in den Wohnimmobilienmarkt durch Analyse der Unsicherheit (Risikos) im Kontext eines Real Business Cycle Modells erklärt werden können. Es wird unterstellt, dass der Wohnimmobiliensektor durch asymmetrische Information charakterisiert ist. Wir zeigen am Beispiel der Vereinigten Staaten, dass diese drastischen Wohnimmobilienpreiszyklen hauptsächlich durch fundamentale ökonomische Faktoren verursacht wurden und Irrationalität (oder Psychologie) lediglich eine beiläufige Rolle einnahm.
\end{abstract}

- JEL Classification: E4, E5, E2, R2, R3

- Keywords: Kreditvergabe, Risikoschocks, Wohnimmobilieninvestmen, Wohnimmobilienpreise

Victor Dorofeenko

Department of Economics and Finance

Institute for Advanced Studies

Stumpergasse 56

A-1060 Vienna, Austria

Gabriel S. Lee (Corresponding Author)

IREBS

University of Regensburg

Universtitaetstrasse 31

93053 Regensburg, Germany

And

Institute for Advanced Studies

Kevin D. Salyer

Department of Economics

University of California

Davis, CA 95616

Contact Information:

Lee: ++49-941.943.5060; E-mail: gabriel.lee@wiwi.uni-regensburg.de

Salyer:(530) 752 8359; E-mail: kdsalyer@ucdavis.edu

${ }^{*}$ Wir danken Tobias Dechant, Ute Koelbl, Kerstin Zeise und besonders Lutz Arnold für hilfreiche Kommentare und Vorschläge, die die Darstellung der Arbeit sowie die Ergebnisse verbessern konnten. 


\section{Einleitung}

"In come the waves: The worldwide rise in house prices is the biggest bubble in history. Prepare for the economic pain when it pops." (The Economist, June 16th 2005). Diese unheilvoll klingende Schlagzeile aus der Zeitschrift The Economist aus dem Jahr 2005 scheint gerechtfertigt, wenn man auf die Entwicklung der globalen Finanz- und Immobilienmärkte in den vergangenen Jahren blickt. Abbildung 1 zeigt den dramatischen Anstieg und den darauf folgenden Rückgang der realen Wohnimmobilienpreise in den Vereinigten Staaten und in einigen ausgewählten europäischen Ländern im Zeitraum von Januar 1999 bis März 2010. ${ }^{1}$

Mit einem Anstieg von 77\% seit Januar 1999 erreichte der U.S. amerikanische Markt für Wohnimmobilien im zweiten Quartal 2007 seinen Höchststand. ${ }^{2}$ Im Vergleich zu den Anstiegen in Spanien (163\%), Großbritannien (117\%) und Irland (153\%), wobei die verschiedenen Wohnimmobilienpreisindices ihren jeweiligen Höchststand zu etwas unterschiedlichen Zeitpunkten erreichten, weist der U.S.- Markt zwar eine relativ moderate Entwicklung auf, bewegte sich aber im Einklang mit diesen Märkten. Nachdem die Wohnimmobilienmärkte in den jeweiligen Ländern gegen Mitte der Dekade ihren Höchststand erreicht hatten, folgte ein plötzlicher Rückgang der Preise für Wohnimmobilien. Zusammen mit diesem signifikanten Einbruch sind in der Literatur mit steigender Häufigkeit Beiträge zu finden, in denen diese starken Schwankungen der Häuserpreise als Bubbles bezeichnet werden oder die starken Anstiege auf irrational exuberance zurückgeführt werden. ${ }^{3}$

Abbildung 1 hier einfügen

\footnotetext{
1 Japan und Deutschland wurden ebenfalls in die Betrachtung aufgenommen, um zu zeigen, dass ein derart deutlicher Anstieg der Häuserpreise nicht in jedem entwickelten Land zu beobachten war.

${ }^{2}$ Der Case-Shiller-Häuserpreisindex weist einen Anstieg von $122 \%$ auf. Der Unterschied zwischen dem Häuserpreisindex des "Office of Federal Housing Enterprise Oversight (OFHEO)" und dem Case-Shiller-Index liegt hauptsächlich in der unterschiedlichen Behandlung teurer Häuser begründet. Der OFHEO-Index berücksichtigt nur Transaktionen, bei denen die entsprechenden Kredite von Institutionen vergeben wurden, die von ihr überwacht werden (Fannie Mae und Freddie Mac). Diese übersteigen jedoch nicht den Betrag von $\$ 417,000$. Der Case-ShillerIndex verfügt über keine derartige Restriktion, was dazu führt, dass teurere Häuser ein höheres Gewicht bei der Indexkonstruktion erhalten.
}

\footnotetext{
${ }^{3}$ Der Ausdruck irrational exuberance wurde wurde erstmals von Alan Greenspan, dem früheren Chairman des "Board of Governors" der Federal Reserve im Jahr 1996 bei einer Aussage vor dem U.S. Kongress verwendet.
} 
Ausdrücke wie Bubbles, Spekulationen am Wohnimmobilienmarkt oder irrational exuberance dominieren im Moment nicht nur die akademische Rhetorik sondern auch die Politik und jegliche Diskussion, in der die derzeitige weltwirtschaftliche Rezession thematisiert wird. Sowohl Akademiker wie Dean Baker und Robert Shiller als auch Politiker wie William Dudley, der Präsident der Federal Reserve Bank von New York, äußern öffentlich, dass der jüngste Anstieg und Zusammenbruch des U.S. Wohnimmobilienmarktes ein gutes Beispiel für eine Blase ist. ${ }^{4}$ Andererseits jedoch gibt es eine Vielzahl renommierter Wissenschaftler, wie bspw. Eugen Fama und John Cochrane ${ }^{5}$, die eine gegenläufige Meinung vertreten. Darüber hinaus deuten einige neue empirische Befunde darauf hin, dass diese drastischen Wohnimmobilienpreiszyklen in den am weitesten fortentwickelten Ökonomien der Welt ${ }^{6}$, insbesondere in den USA, hauptsächlich durch fundamentale ökonomische Faktoren verursacht wurden und Irrationalität (oder Psychologie) lediglich eine beiläufige Rolle einnahm.

Selbst unter Wissenschaftlern existieren somit unterschiedlichste Meinungen bzgl. dieser Thematik; woher soll man nun also wissen, ob diese starken Preisanstiege durch fundamentale ökonomische Prinzipien, also Angebot und Nachfrage, verursacht wurden oder durch Irrationalität bzw. psychologische Aspekte? Eine Definition, die von Medien und Praktikern gleichermaßen verwendet wird, um eine Blase zu definieren, stammt aus dem Palgrave's Dictionary of Political Economy (1926): "Any unsound undertaking accompanied by a high degree of speculation". Eine andere Definition, welche häufig in der Wissenschaft Anwendung findet ist "that part of asset price movement that is unexplainable based on what we call fundamentals. Fundamentals are a collection of variables that we believe should drive asset prices." (Garber, 2000) ${ }^{7}$ Ähnlich wie Stiglitz (1990) ist

\footnotetext{
4 Nachzulesen in kürzlich erschienenen Publikationen wie "The Menace of an Unchecked Housing Bubble," (2006) von Dean Baker in The Economists' Voice, "Why We'll Always Have More Money Than Sense" in Newsweek (December 30, 2009) und "Unlearned Lessons from the Housing Bubble" in The Economists' Voice (July 2009) by Shiller. Ebenso in der Rede "Asset Bubbles and the Implications for Central Bank Policy" (April 7, 2010) gehalten von Dudley vor dem Economic Club of New York.

5 Nachzulesen in einem Interview von John Cassidy mit Eugene Fama und John Cochrane unter dem Titel "Rational Irrationality" in der Ausgabe des New Yorker vom 13. Januar 2010.

6 Mit Ausnahme von Deutschland, der Schweiz und Japan.

7 Stiglitz (1990, pg. 13) verwendet eine Definition, die der von Garber (2000) änlich ist und die ebenfalls häufig in der akademischen Literatur verwendet wird: "if the reason that the price is high today is only because investors believe that the selling price is high tomorrow - when 'fundamental' factors do not seem to justify such a price then a bubble exists. At least in the short run, the high price of the asset is merited, because it yields a return
} 
Shiller (1989) grundsätzlich an der Bepreisung von Assets interessiert "...can we trace the sources of movements back in a logical manner to fundamental shocks affecting the economy? Or are price movements due to changes in opinion or psychology, that is, changes in confidence, speculative enthusiasm ... that are best thought of coming ultimately from peoples minds?"

Dieser Artikel verfolgt daher zwei Ziele. Zum einen beschäftigen wir uns mit der Frage, die Shiller (1989) aufwirft und beantworten diese: Können Wohnimmobilienpreisdynamiken durch Schocks fundamentaler ökonomischer Variablen erklärt werden? Wir stellen dazu zunächst einige aktuelle empirische Arbeiten vor, die sich mit dem Einfluss fundamentaler ökonomischer Faktoren auf Wohnimmobilienpreiszyklen beschäftigen und diese zu erklären versuchen. ${ }^{8}$ Zum anderen beschreiben wir ein Modell (Dorofeenko, Lee and Salyer (2011), im Weiteren als DLS bezeichnet), das in der Lage ist, die wichtigste Komponente der derzeitigen Finanzkrise, nämlich Änderungen im Risiko des Wohnimmobilienmarktes, darzustellen. Darüber hinaus bilden wir die Analyse von Risikoschocks in dem Modell ab, das zwei sehr charakteristische Merkmale des Wohnimmobilienmarktes berücksichtigt: (i) Wohnimmobilenpreise sind deutlich volatiler als der volkswirtschaftliche Output. (ii) Investments in den Wohnimmobilienmarkt sind im Vergleich zu Investments in andere Sektoren etwa doppelt so volatil. Diese beiden bekannten, marktspezifischen Gegebenheiten wurden bisher häufig mit Wohnimmobilienpreiszyklen in Verbindung gebracht. ${ }^{9}$

Abbildung 2 hier einfügen

Plausible Kalibrierungen dieses Modells in Bezug auf sich im Zeitablauf verändernde Unsicherheit (Risiko) liefern laut DLS (2011) sowohl qualitativ als auch quantitativ aussagekräftige Ergebnisse und betonen die Rolle dieser Unsicherheit im Verlauf des Wohnimmobilienpreis- und (capital gain plus dividend) equal to that of alternative assets."

8 Da wir nicht über die Heterogenität von Konsumenten (Investoren) in Bezug auf ihre Erwartungen an die langfristigen ökonomischen Gegebenheiten sprechen, behandeln wir nicht explizit die Thematik der Wohnimmobilienpreisblasen. Für entsprechende Literatur wird auf Piazzesi und Schneider (2009) verwiesen, die zeigen, dass eine kleine Anzahl optimistischer Individuen großen Einfluss auf die Preise für Wohnimmobilien haben kann, da eben diese Individuen die marginalen Händler/Käufer darstellen.

9 Abbildung 2 und Tabelle 2 stellen diese zyklischen und statistischen Besonderheiten im Zeitraum zwischen 1975 und Q2 2007 dar. 
Wirtschaftszyklus. So vergleichen DLS (2011) bspw. die Impulsresonanzfunktion aggregierter Variablen (BIP, Konsumausgaben und Investitionen) bei einem einprozentigen Anstieg von Technologieschocks, die sich auf den Bausektor auswirken, zu einem einprozentigen Anstieg von Risikoschocks, die die Häuserproduktion beeinflussen. DLS (2011) finden heraus, dass die Auswirkungen von Risikoschocks fast so umfangreich sind wie die Auswirkungen von Technologieschocks. Dieser Vergleich ist auf Wohnimmobilienmarktvariablen wie den Preis von Wohnimmobilien und das Investitionsvolumen übertragbar. Weitaus wichtiger ist jedoch, dass das Modell die Möglichkeit bietet, Wohnimmobilienpreise und Schwankungen der Investitionen in den Wohnimmobilienmarkt rational zu erklären. Da sich das Modell lediglich mit der Angebotseite beschäftigt und andere Charakteristika des Wohnimmobilienmarktes nicht mit in Betracht zieht, kann es in diesem Sinne als nicht vollständig bezeichnet werden Nichtsdestotrotz sind wir der Ansicht, dass der hier vorgestellte Ansatz dazu beitragen kann, die Auswirkungen zeitvariablen Risikos auf den Wohnimmobilienmarkt, den Finanzierungsmarkt für Wohnimmobilien sowie Wohnimmobilienmarktzyklen zu untersuchen, ohne dabei auf Argumente wie Irrationalität oder auf psychologisch erklärte Verhaltensweisen von Konsumenten und Investoren zurückgreifen zu müssen.

\section{Wohnimmobilienpreise und deren ökonomische Determi-}

\section{nanten}

In diesem Abschnitt stellen wir einige aktuelle empirische Arbeiten vor, die sich mit dem Einfluss fundamentaler ökonomischer Variablen auf Wohnimmobilienmarktzyklen beschäftigen. Zusammenfassend kommen diese Studien zu dem Ergebnis, dass günstige Finanzierungsbedingungen aufgrund niedriger Hypothekenzinsen, höhere Einkommen und gestiegene Produktivität, Finanzinnovationen sowie die Liberalisierung auf dem Gebiet der Finanzierung von Wohnimmobilien und die demographische Entwicklung die Nachfrage in den meisten entwickelten Ländern stimuliert haben. Eine restriktive Politik bei der Ausweisung von Bauland hat zumeist verhindert, dass 
diese Nachfrage befriedigt werden konnte, was den Anstieg der Wohnimmobilienpreise zusätzlich verstärkte.

\subsection{Zinsen und Nutzungskosten}

Bei der Bewertung von Aktien wird das Verhältnis zwischen dem Aktienkurs und der Dividende häufig als Indikator dafür verwendet, um eine Einschätzung darüber abzugeben, ob die Aktie über- oder unterbewertet ist. Analog dazu dient das Verhältnis zwischen dem Preis einer Wohnimmobilie und der impliziten Miete, ${ }^{10}$ die der Eigentümer erhält (spart), wenn er die Immobilie bewohnt, häufig als ein Indikator für die aktuellen Gegebenheiten am Wohnimmobilienmarkt. Da diese Kennzahl bei längerfristiger Betrachtung zu einem langfristigen Durchschnitt tendiert, wird jede starke Abweichung von diesem langfristigen Durchschnitt als Anzeichen für eine Über- bzw Unterbewertung von Wohnimmobilien interpretiert.

Poterba (1984), Hubbard und Mayer (2009) und Himmelberg et al. (2005) zeigen jedoch, dass die Interpretation dieser Kennzahl oftmals eine Überbewertung impliziert. Ihr Ansatz, die Nutzungskosten eines Hauses heranzuziehen, führt zu modifizierten Ergebnissen. Sie argumentieren, dass das Verhältnis zwischen Miete und Preis einer Wohnimmobilie von den Hypothekenzinsen, der erwarteten Inflation, der erwarteten Wertsteigerung der Immobilie sowie der Steuerlast, die mit der Immobilie verbunden ist, abhängig sein sollte. Entsprechend ihres Ansatzes sollte die Miete im Gleichgewicht den Nutzungskosten der Immobilie entsprechen. Dies lässt sich formal folgendermaßen darstellen:

$$
R=P(i+\tau+m-g)
$$

wobei $R$ die Miete und $P$ den nominalen Preis der Wohnimmobilie darstellt. $i$ repräsentiert den nominalen nach-Steuer-Hypothekenzinssatz, $\tau$ eine Steuer für selbst genutztes Wohneigentum, $m$ die Instandhaltungskosten sowie Abschreibungen und $g$ die erwartete Wertsteigerung der Wohn-

\footnotetext{
10 Eine weitere häufig gebrauchte Kennzahl, um den Wert eines Hauses zu evaluieren, ist das Verhältnis zwischen dem Einkommen und der impliziten Miete zu bilden. Diese Kennzahl stellt eine Einschätzung der Erschwinglichkeit des Gutes Wohnen dar.
} 
immobilie. Hubbard und Mayer (2009) sowie Himmelberg et al. (2005) betonen jedoch den starken Zusammenhang zwischen Zinsen und Wohnimmobilienpreisen in den Vereinigten Staaten. Daher sollte das Verhältnis von Wohnimmobilienpreisen zu den impliziten Nutzungskosten zur Bewertung von Wohnimmobilienpreisen mit Vorsicht interpretiert werden.

Abbildung 3 (entnommen von Andre (2010)) zeigt das Verhältnis von tatsächlichen Wohnimmobilienpreisen zur Miete sowie die Nutzungskosten, wobei zur Berechnung der Hypothekenzins und die Rendite von Staatsanleihen verwendet wurden. Die Grafik zeigt deutlich, dass ein Rückgang des Zinsniveaus einen signifikanten Anteil der Wohnimmobilienpreisanstiege in den meisten Ländern zwischen 2000 und 2004 erklären kann. Glaeser, Gottlieb und Gyourko (2010) bezweifeln jedoch diese wichtige Funktion der Zinsen bei der Erklärung der momentanen U.S. Wohimmobilienmarktzyklen. Sie zeigen, dass Zinsen nur einen kleinen Teil der Volatilität in Regionen erklären können, in denen das Angebot an neuen Wohnimmobilien beschränkt ist.

Abbildung 3 hier einfügen

\subsection{Produktivitätssteigerungen}

Eine weitere aktuelle Studie von Kahn (2009) zeigt, dass der Anstieg der Wohnimmobilienpreise in den Vereinigten Staaten zum überwiegenden Teil auf fundamentale ökonomische Faktoren, insbesondere auf erwartete Produktivitätssteigerungen (und somit Einkommenssteigerungen), zurückzuführen ist. Kahn (2009) entwickelt ein neoklassisches Wachstumsmodell, in dem Bauland und Wohnen als Faktoren implementiert werden, und welches einen Großteil der Wohnimmobilienpreisschwankungen sowie den Zeitpunkt des Auftretens dieser Schwankungen über die vergangenen 40 Jahre hinweg erklären kann. Kahn (2009) gelangt zu der Erkenntnis, das der vehemente Anstieg der Wohnimmobilienpreise seit Mitte der 90er Jahre bis 2007 hauptsächlich auf die weit verbreitete Meinung zurückzuführen ist, dass weiteres Produktivitätswachstum zu weiterem Einkommenswachstum führen würde. Als man 2007 wahrnahm, dass sich das Produktivitätswach- 
stum verlangsamte, führte dies zu einem dramatischen wirtschaftlichen Abschwung, was auch den Wohnimmobilienpreisboom deutlich drosselte und die Ausfallrate von Hypotheken erhöhte. Als logische Konsequenz setzt sich der bis dahin konstante Anstieg der Wohnimmobilienpreise auch nicht mehr fort. Laut Kahn (2009) setzte die Verlangsamung des U.S. Produktivitätswachstums bereits im Jahr 2004 ein. Diese Verlangsamung wurde jedoch erst im Jahr 2007 wahrgenommen. Daher war es, basierend auf der ökonomischen Datenlage im Jahr 2004, durchaus rational, gestiegene Wohnimmobilienpreise für kurze Zeit noch als gerechtfertigt anzusehen, ehe man 2007 realisierte, dass sich das Produktivitätswachstum tatsächlich nachhaltig verlangsamte.

\subsection{Finanzinnovationen und Liberalisierungen bei der Finanzierung von Wohnimmobilien}

Faviluki et al.(2010) führen Belege dafür an, dass der jüngste Auf- und Abschwung (bspw. dargestellt anhand der Schwankungen des Mietpreis/Kaufpreis-Verhältnisses in den USA) auf Liberalisierungen bei der Finanzierung von Wohnimmobilien zurückzuführen ist. Zu solchen zählen bspw. die Lockerung der Anforderungen an den Kreditnehmer bei der Aufnahme eines Kredits (keine umfangreichen Sicherheiten), sinkende Fremdkapitalkosten oder rückläufige Transaktionsgebühren beim Kauf einer Immobilie. In einer Simulation, in der der Zeitraum von 2000 bis 2009 analysiert wird, gibt ihr Modell das Ansteigen des U.S. Mietpreis/Kaufpreis-Verhältnisses von 2000 bis 2006 wieder, was auch in der Realität zu beobachten war. Darüber hinaus wird ab dem Jahr 2007 ein deutlicher Rückgang der Wohnimmobilienpreise vorhergesagt. Eine ähnliche Studie von Sommer, Sullivan and Verbrugge (2010) zeigt ebenfalls, dass ein Rückgang der Finanzierungskosten, gelockerte Finanzierungsstandards und höhere Einkommen etwa 50\% des Anstiegs des U.S Mietpreis/KaufpreisVerhältnisses zwischen 1995 und 2005 erklären können. 


\subsection{Die demographische Entwicklung}

Spanien und Irland hatten in den vergangenen zehn Jahren die höchsten Wohnimmobilienpreisanstiege zu verzeichnen. Daher werden diese beiden Länder oftmals als klassisches Beispiel für eine Wohnimmobilienpreisblase angeführt. Garriga (2010) zeigt jedoch, dass die demographische Entwicklung, Zinsen sowie restriktive Baulandpolitik zusammen 87\% der Preissteigerungen spanischer Wohnimmobilien von 1995 bis 2007 erklären können. Der demographische Faktor alleine erklärt $52 \%$, der Rückgang der Hypothekenzinsen von $4 \%$ auf $3 \%$ sogar $60 \%$ der Preissteigerungen. Das Modell zeigt auch, dass der Häuserpreisboom sich um 25\% verringert hätte, wenn der Hypothekenzinssatz 200 Basispunkte (2\%) höher gelegen hätte. Darüber hinaus sagt das Modell voraus, dass bei den derzeitigen Hypothekenzinsen, der momentanen Arbeitslosigkeit und der niedrigen Produktivität die Wohnimmobilienpreise zwischen $24 \%$ und $29 \%$ sinken müssten. Diese Vorhersage stimmt mit den derzeitigen spanischen Daten überein.

\subsection{Restriktives Wohnimmobilienangebot und rationale Bubbles}

Das deutliche Absinken der Wohnimmobilienpreise kann auch durch die Inelastizität des Angebots an Wohnimmobilien verursacht werden. Laut Kahn (2008) sowie Glaeser, Gyourko und Saiz (2008) ist es aufgrund der relativ geringen Elastizität des Angebots an Wohnimmobilien möglich, dass Hauspreise in Zeiten überdurchschnittlichen ökonomischen Wachstums stärker steigen als die Einkommen und somit auch deutlicher fallen, wenn sich das Wachstum verlangsamt. Darüber hinaus zeigen Glaeser, Gyourko und Saiz (2008), dass Informationen über grundlegende ökonomische Faktoren tendenziell eher die Wohnimmobilienpreise beeinflussen, als Investitionen in den Wohnimmobiliensektor, je geringer die Elastizität des Angebots an derartigen Investitionen ist. Ihr Modell zeigt, dass rationale Blasen existieren können, wenn das Angebot an Wohnimmobilien fix ist, jedoch nicht, wenn das Angebot elastisch ist und eine gewisse Anzahl an potentiellen Käufern existiert. Folglich impliziert die Literatur, die sich mit der Angebotsrestriktion von Wohnim- 
mobilien befasst, dass die daraus folgenden, erweiterten Erkenntnisse bzgl. der Reaktion auf Veränderungen fundamentaler ökonomischer Variablen, eine ähnlich wichtige Stellung einnehmen wie die kürzlich beobachteten Wohnimmobilienmarktzyklen.

\section{Risikoschocks und Immobilienmärkte von DLS (2011)}

Dieser Abschnitt befasst sich mit dem zweiten Ziel dieses Artikels und präsentiert den Bezugsrahmen und die empirischen Ergebnisse von DLS (2011). Sie analysieren die Rolle sich im Zeitablauf ändernder Unsicherheit (Risikoschocks) in einem multisektoralen Real Business Cycle Modell, welches die Errichtung von Wohnimmobilien (entwickelt von Davis und Heathcote (2005)) und einen Finanzsektor mit Kreditvergabe unter asymmetrischer Information (z.B. Carlstrom und Fuerst (1997, 1998); Dorofeenko, Lee und Salyer (2008)) einschließt. Sie modellieren Risikoschocks als Mittel, um einen Spread in der Verteilung der Technologieschocks zu erhalten, welche die Errichtung von Wohnimmobilien beeinflussen, und untersuchen, wie Änderungen in der Unsicherheit die Gleichgewichtscharakteristika beeinflussen. ${ }^{11}$

Zu diesem Zweck nutzen DLS (2011) das Davis und Heathcote (2005) WohnungimmobilienModell, welches, wie von den Autoren gezeigt, die hohe Volatilität reproduzieren kann, die in Bauinvestitionen beobachtet wird, obwohl in der Ökonomie keine Friktionen auftreten. Die neue Analyse in Christiano u.a. (2008) liefert jedoch zwingende Belege, dass finanzielle Friktionen eine wichtige Rolle in Konjunkturzyklen spielen. Unter Anbetracht der aktuellen Finanzkrise scheint es begründet, diese Rolle in Kombination mit einem Immoiliensektor zu untersuchen. ${ }^{12}$ Folglich modifizieren wir die Analyse von Davis und Heathcote (2005), indem wir einen Finanzsektor

\footnotetext{
11 Einige der jüngsten Arbeiten, die auch das Wohnungs- und Kreditwesen untersuchen, sind: Iacoviello und Minetti (2008) und Iacoviello und Neri (2010), in welchen ein neu-keynesianisches DGSE-Modell mit zwei Sektoren in der empirischen Analyse verwendet wird. Iacoviello (2005) analysiert die Rolle, welche Immobilien-Sicherheiten für die Geldmarktpolitik haben und Aoki, Proudman und Vliegh (2004) analysieren Hauspreis-Verstärkungseffekte in Konsum und Wohnungsinvestitionen über den Konjunkturzyklus. Keine dieser Analysen verwendet Risikoschocks als einen Impulsmechanismus.

12 Christiano u.a. (2008) verwenden ein neu-keynesianisches Modell, um den relativen Einfluss von Schocks zu analysieren, welche im Arbeits- und Gütermarkt, in der Geldmarktpolitik und im Finanzsektor auftreten. Sie finden heraus, dass zeitvariante zweite Momente, d.h. Risikoschocks, relativ zu den anderen Impulsmechanismen quantitativ wichtig sind.
} 
zur Ökonomie hinzufügen und voraussetzen, dass Bauunternehmen ihre zu erbringende Leistung durch Bankenkredite finanzieren müssen. Diese Modifikation erweist sich als wichtig; wir zeigen zum Beispiel, dass durch das Einbeziehen eines expliziten Finanzmarktes in dieses Modell große Bewegungen in den Hauspreisen dargestellt werden, ein Merkmal der Daten, welches in der Analyse von Davis und Heathcote (2005) fehlt. Wir zeigen auch, dass die Hauspreise in unserem Modell durch erwartete Bankrotte und die damit verbundenen Agenturkosten beeinflusst werden; diese stellen einen endogenen, zeitvarianter Aufschlagsfaktor dar, der den Hauspreis beeinflusst. Die Volatilität dieses Aufschlags übersetzt sich in erhöhte Volatilität in den Hauspreisen. Überdies impliziert das Modell, dass dieser endogene Aufschlag auf Wohnen genauso wie die mit den Krediten im Immobiliensektor verbundene Risikoprämie antizyklisch sein sollte; beide Merkmale sind in den Daten zu beobachten.

\subsection{Eine kurze Beschreibung des Modells}

Wie oben bemerkt, begründet sich das Modell von DLS (2011) auf zwei getrennte Literaturstränge: Davis und Heathcotes (2005) multisektorales Wachstumsmodell mit Immobilien und Dorofeenkos, Lees und Salyers (2008) Kreditkanal-Modell mit zeitvarianter Unsicherheit. Die Ökonomie besteht aus zwei Agenten, einem Konsumenten und einem Unternehmer und aus fünf Sektoren: einem Zwischenproduktsektor, einem Endproduktsektor, einem Immobiliengütersektor, einem Bankensektor und dem Arbeitsmarkt. Der Zwischenproduktsektor besteht aus drei in vollkommener Konkurrenz zueinander stehenden Industrien: einem Bau-/Konstruktionssektor, einem Fertigungssektor und einem Dienstleistungssektor. Der Output dieser Sektoren wird dann zusammengefasst, um ein Investitionsgut und ein Konsumgut, welches konsumiert oder als Kapitalinvestition verwendet werden kann, zu produzieren; diese Sektoren stehen auch in vollkommener Konkurrenz zueinander. Unternehmer kombinieren Investitionen mit einem festen Faktor (Land), um Immobilien zu produzieren; in diesem Sektor spielen der Kreditkanal und die Finanzintermediation eine Rolle.

\section{Firmen}


Zuerst betrachten wir den Zwischenproduktsektor, in dem die repräsentative Firma in jedem Sektor durch eine Cobb-Douglas Produktionsfunktion mit konstanten Skalenerträgen und mit Kapital und Arbeit als Inputs charakterisiert wird. Diese Produktionsfunktion wird beeinflusst von einem arbeitsverstärkenden Produktivitäts- (Technologie-) Schock. ${ }^{13}$ Diese intermediären Firmen maximieren eine konventionelle statische Gewinnfunktion in jeder Periode.

Die Zwischenprodukte werden dann als Inputs verwendet, um die beiden Endprodukte zu produzieren: Konsum/Kapitalinvestitionen und Immobilieninvestitionen. Diese Produktionstechnologie entspricht ebenfalls einer Cobb-Douglas-Funktion mit konstanten Skalenerträgen. Mit den Zwischenprodukten als Inputs lösen die Firmen des Endproduktsektors ein statisches Gewinnmaximierungsproblem zum Zeitpunkt $t$, wobei der Preis des Konsumgutes auf 1 normiert wird. Neue Immobilien werden schließlich von den Unternehmern (d.h. Immobilienentwicklern) unter Verwendung des Investitionsgutes Wohnimmobilien und von Land als Inputs produziert. Für einen Unternehmer (Immobilienentwickler) wird angenommen, dass die Produktionsfunktion konstante Skalenerträge mit einem idiosynkratischen Technologieschock aufweist.

In jeder Periode wird die Produktion der neuen Wohnimmobilien zum Bestand der abgeschriebenen Wohneinheiten hinzugefügt. In Anbetracht des Fehlens von aggregierter Unsicherheit in der neuen Wohnimmobilienproduktion ist die effektive pro-Kopf-Wohneinheit die Summe der Anzahl an abgeschriebenen Wohneinheiten der Vorperiode plus die Gesamtanzahl an Wohnimmobilienproduktion minus den Verlust auf Grund von Bankrotten eines Teils der Immobilienentwickler. Der letzte Faktor ist kritisch und wird detaillierter in der Diskussion des Credit Channel diskutiert, welche unten dargelegt wird. Überdies unterliegt die Gesamtanzahl der Wohnimmobilienproduktion Investitionsanpassungskosten, wie sie in Christiano u.a. (2005) verwendet werden. ${ }^{14}$

\footnotetext{
13 Es ist zu beachten, dass mit dem arbeitsverstärkenden Technologieschock $Y=f(K, z L)$, wobei $Y$ den Output und $K, z$ und $L$ das Kapital, den Technologieschock und Arbeit bezeichnen, wie permanente Schocks auf die Kapitalintensität ( $\frac{K}{L}$, welche die effiziente Einheit misst) einen permanenten Effekt auf den Pro-Kopf-Output ( $\left.\frac{Y}{L}\right)$ haben wird.

14 Unter Annahme von quadratischen Anpassungskosten (QAC) sind große Investitionen mit sehr hohen Anpassungskosten verbunden. Somit hat die Firma einen Anreiz, eine gegebene Anpassung des Kapitalstocks über mehrere Perioden auszudehnen. Wir verwenden Christiano u.a. (2005), da diese zeigen, dass das aggregierte Verhalten der Investitionen besser ausgewiesen wird durch eine Spezifikation, welche die Änderungen in den Investitionen bestraft.
} 


\section{Haushalte}

Es gibt zwei Typen von Haushalten: Unternehmer (die auch die Besitzer der Firmen sind, welche Häuser produzieren und externe Finanzierung benötigen) und Haushalte, die Darlehen an die Finanzintermediäre anbieten. Um Eigenfinanzierung durch den Unternehmer auszuschließen (d.h. dies würde das Vorhandensein von Agentenkosten beseitigen), wird angenommen, dass der Unternehmer die zukünftigen Cashflows mit einer schnelleren Rate diskontiert als der Haushalt.

Der repräsentative Haushalt zieht in jeder Periode Nutzen aus Konsum, Wohnimmobilie und Freizeit; alle diese Daten werden pro Kopf gemessen. Der unmittelbare Nutzen für jedes Mitglied des Haushalts wird durch eine Cobb-DouglasFunktion definiert. Der Haushalt maximiert den erwarteten Nutzen über seine Lebenszeit unter der Budgetbeschränkung, die besagt, dass die Agenten in jeder Periode das Arbeitseinkommen mit dem Einkommen der Assets (Kapital, Wohnimmobilie, Land und Darlehen an den Bankensektor) kombinieren und dazu verwenden, Konsum, neuen Wohnungsbau und Investitionen zu erwerben. Das heißt, die Haushalte fällen ihre Entscheidungen über Arbeit, Konsum und Sparen/Investieren. Die Unternehmer maximieren ebenfalls ihren erwarteten Nutzen über die Lebenszeit mit ihrer entsprechenden Budgetbeschränkung.

\section{Der Kreditkanal: Housing Entrepreneurial Contract}

Die Ökonomie, welche oben beschrieben wurde, ist identisch zu der, welche in Davis und Heathcote (2005) untersucht wurde, abgesehen von den hinzugefügten Risikoschocks und den Finanzintermediären, die die Wohnimmobilienproduktion beeinflussen. ${ }^{15}$

Die Ökonomie unterstellt, dass ein Kontinuum von Wohnimmobilien produzierenden Firmen der Masse 1 im Besitz von risikoneutralen Unternehmern (Entwicklern) sind. Mit dem Sparkapital der Haushalte bieten die Finanzintermediäre Kredite an die Unternehmer über den optimalen Finanzvertrag. Der Vertrag ist definiert durch den Umfang des Kredits und einen cutoff Produktionswert für den idiosynkratischen Technologieschock des Unternehmers. Unternehmer verwenden ihren Nettobesitz und Kredite von den risikoneutralen Finanzintermediären um die Faktoren

\footnotetext{
${ }^{15}$ Zudem lassen wir, wie oben angemerkt, Steuern und Staatsausgaben außer Acht.
} 
(Inputs) für die Wohnimmobilienproduktion zu erwerben. Die Menge an Faktoren (Wohnungsinvestitionen und Land) wird bestimmt und bezahlt bevor der idiosynkratische Technologieschock bekannt ist.

In Anbetracht der Realisierung des idiosynkratischen Schocks eines jeden der Unternehmer (Entwickler) auf die Wohnimmobilienproduktion werden einige Immobilienentwickler nicht in der Lage sein, ihre Kreditzahlungen zu erfüllen und werden bankrott gehen. ${ }^{16}$ Das heißt, wenn der idiosynkratische Schock größer ist als der cutoff Punkt des Darlehens, dann ist der Unternehmer solvent und der Kredit von der Bank wird zurückgezahlt; ansonsten erklärt der Unternehmer Bankrott und die Produktion wird von den Finanzintermediären zu Kosten überwacht, die proportional zu den totalen Faktorzahlungen sind. Die Banken übernehmen die Transaktionen dieser insolventen Firmen, müssen aber eine Agenturgebühr zahlen. ${ }^{17}$. Diese Agenturgebühren beeinflussen demzufolge die aggregierte Produktion von Wohnimmobilien und implizieren einen endogenen Aufschlag auf die Hauspreise. Das heißt, da ein Teil des Wohnimmobilienoutputs an die Agenturgebühren verloren wird, muss der Preis von Wohnimmobilien erhört werden, um die Faktor- (Input) Kosten zu decken.

\section{Finanzintermediäre}

Die Banken im "Bankensektor" des Modells agieren als risikoneutrale Finanzintermediäre, die im Gleichgewicht keinen Gewinn erzielen. Das ist eine klare Rolle für "Banken" in dieser Ökonomie, da durch Zusammenlegen die gesamte aggregierte Unsicherheit der Wohnimmobilienproduktion beseitigt werden kann. Der Bankensektor erhält Einlagen von den Haushalten und diese werden durch Gelder aus zwei Quellen zurückgezahlt: Darlehensrückzahlung von solventen Wohnimmobilienproduzenten und der Wert des Wohnimmobilienoutputs, abzüglich der Überwachungskosten, von insolventen Wohnimmobilienfirmen.

\footnotetext{
${ }^{16}$ Auf der anderen Seite wären Unternehmer, die einen positiven idiosynkratischen Schock beziehen, solvent und würden ihre Konsumwahl treffen; diese bestimmt zum Teil ihren Nettobesitz für die nächste Periode und führt ihre Wohnimmobilienproduktion fort.

17 Man kann diese Agenturkosten (Gebühren) auch als Einmalkosten betrachten, welche von der Bank übernommen werden, um die Produktion der insolventen Firmen zu erledigen.
} 


\section{Schocks}

Es gibt vier verschiedene Schocks in dieser Ökonomie: drei sektorale Produktivitäts-TechnologieSchocks für Konstruktion, Herstellung und Dienstleistungen und einen idiosynkratischen Technologieschock der Unternehmer, welcher die Wohnimmobilienproduktion beeinflusst. Alle diese Schocks folgen einem autoregressiven Prozess erster Ordnung (d.h. AR(1)).

\subsection{Empirische Ergebnisse}

\subsubsection{Kalibrierung und Daten ${ }^{18}$}

Das Modell wird durch eine standardmäßige log-Linearisierungsmethode gelöst, welche oft in der Literatur zur stochastischen allgemeinen Gleichgewichtstheorie angewendet wird. Eine hohe Motivation, das Modell von Davis und Heathcote (2005) und das Modell von DLS (2011) zu verwenden, besteht darin, dass die theoretischen Konstrukte empirische Pendants haben. Hier können die Modellparameter zu den Daten kalibriert werden. Wir verwenden direkt die Parameterwerte, die von den o.g. Autoren gewählt wurden; die Leser werden für eine Erklärung ihrer Kalibrierungsmethode auf ihre Artikel verwiesen. ${ }^{19}$.

Vor der Diskussion der empirischen Ergebnisse stellen wir folgende statistische Merkmale der U.S. Wirtschaft dar. Abbildung 3 zeigt die zyklischen und statistischen Merkmale für die Periode von 1975 bis einschließlich des zweiten Quartals von 2007. Wie in der Einleitung bemerkt sind die beiden wohlbekannten Charakteristika einfach. i) Hauspreise sind viel volatiler als der Output und ii) Investitionen in Wohnraum sind fast doppelt so volatil wie Investitionen in gewerblich genutzte Gebäude. ${ }^{20}$

\footnotetext{
18 Die Ergebnisse dieser Kalibrierung unterscheiden sich etwas von denen von DLS (2011), weil wir Daten von 1997 bis 2007 verwenden, wohingegen DLS (2011) den Erhebungszeitraum 1948 bis 2007 analysieren.

19 Der Parameter der Anpassungskosten wird in Christiano u.a. (2005) für die Quartalsdaten auf 3 gesetzt. DLS (2011) verwenden den relativ niedrigen Wert 0,02 für den Parameter der Anpassungskosten, da ihr Modell auf jährlicher Frequenz beruht. Überdies zeigen DLS (2011), dass nur ein geringer Anteil der Anpassungskosten benötigt wird, damit ihr Finanz-Kredit-Modell auf diverse stilisierte Fakten passt.

20 Andere Ergebnisse für aggregierte Variablen zu Geschäfts- und Wohnimmobilien siehe Dorofeenko, Lee und Salyer (2010).
} 
Table 1: Standardabweichungen im Verhältnis zum BIP für eine wenig volatile Ökonomie

\begin{tabular}{|c|c|c|c|}
\hline $\begin{array}{l}\text { Variablen } \\
\text { (im Verhältnis zum BIP) }\end{array}$ & $\begin{array}{l}\text { DLS }(2011) \\
(1997-2007)\end{array}$ & \multirow[t]{2}{*}{$\begin{array}{l}(\mathrm{D} \& \mathrm{H}) \\
(1948-2001)\end{array}$} & \multirow[t]{2}{*}{$\begin{array}{l}\text { Daten }\left({ }_{-}^{+} 2 \mathrm{SD}\right) \\
(1997-2007)\end{array}$} \\
\hline Volatilität der Unsicherheit & wenig volatile Ökonomie bei 0.32 & & \\
\hline $\begin{array}{l}\text { Investitionen in Gewerbeimmobilien } \\
\text { Investitionen in Wohnimmobilien } \\
\text { Hauspreis }\end{array}$ & $\begin{array}{l}4.89 \\
\mathbf{5 . 1 9} \\
\mathbf{1 . 6 1}\end{array}$ & $\begin{array}{l}3.21 \\
6.12 \\
0.4\end{array}$ & $\begin{array}{l}2.3( \pm 1.03) \\
5.04( \pm 2.25) \\
1.86( \pm 0.83)\end{array}$ \\
\hline
\end{tabular}

\subsubsection{Zweite Momente}

Aufgrund des Ziels dieses Artikels fokusieren wir unser Hauptinteresse auf: die Eigenschaften der zweiten Momente des Wohnimmobilienzyklus unter Verwendung des DLS (2011) Modells. ${ }^{21}$

Die Ergebnisse dieses Modells werden in Tabelle 1 gezeigt. Wir vergleichen die zweiten Momente der DLS (2011) Ökonomie mit denen des Davis und Heathcote (2005) Modells und mit den Daten. Für den letzteren Vergleich stellen wir einen Bereich von zwei Standardabweichungen als grobes Maß eines 95\% Konfidenzintervalls dar.

Wenn die stochastische Volatilität eines Unsicherheitsparameters für den Wohnimmobiliensektor festgesetzt wird um eine wenig volatile Ökonomie zu simulieren, repliziert das Modell im Grunde die groben Merkmale, die in den Daten zu sehen sind: die Volatilitäten für Hauspreise und Investitionen in Wohnimmobilien. Überdies zeigt das DLS (2011) Modell, dass die Anpassungskosten für die Investitionen ein wichtiges Merkmal für den Abgleich beider vorher erwähnter stilisierter Fakten sind. Ohne die Anpassungskosten für die Investitionen könnte man immer noch die Volatilität der Hauspreise abgleichen, indem man unrealistisch hohe Parameter für den Risikoschock verwendet. Aber der Erfolg in dieser Dimension geht auf Kosten der Volatilität von Investitionen in Wohnimmobilien, welche über fünf Mal größer ist als in den Daten beobachtet. ${ }^{22}$

\footnotetext{
21 DLS (2011) produzieren Gleichgewichtswerte, welche weitgehend mit den Daten übereinstimmen. Überdies werden die zeitgleichen Korrelationen und die lead-lag Struktur etlicher weiterer entscheidender Geschäfts- und Wohnvariablen sorgfältig in DLS (2011) diskutiert.

22 Davis und Heathcote (2005) können ohne Friktionen nur die Volatilität der Investitionen in Wohnimmobilien anpassen.
} 


\subsubsection{Dynamik: Resonanzfunktionen}

Um zu analysieren wie der Mechanismus zur Darlehensgewährung (Kredit) die Effekte eines Risikoschocks beeinflusst, analysieren wir die Resonanzfunktionen des Modells, wobei der Parameter der Überwachungs- (Agentur-) Kosten auf 0,25 gesetzt wird. ${ }^{23}$ Ohne Überwachungskosten sollten die Risikoschocks das Verhalten der Hauspreise und der Investitionen in Wohnimmobilien nicht beeinflussen. Wir untersuchen darüber hinaus die Antwort der Ökonomie auf eine Innovation in der Produktivität im Bausektor (wobei dies der wichtigste Input für das Wohnimmobilieninvestitionsgut ist). Die Resonanzfunktionen (auf eine Innovation von $1 \%$ bei beiden Schocks) für eine ausgewählte Menge an wichtigen Variablen werden in den Abbildungen 4 - 5 dargestellt. ${ }^{24}$

Zuerst wenden wir uns dem Verhalten der drei wichtigsten makroökonomischen Variablen zu, nämlich BIP, Konsum der Haushalte (bezeichnet mit PCE) und Arbeit (Stunden), dargestellt in Abbildung 4.

\section{Abbildung 4 hier einfügen}

Die Resonanz bzgl. eines positiven Technologieschocks auf den Konstruktionssektor hat den vorhergesagten Effekt, dass alle drei nämlich, BIP, Konsum und Arbeit ansteigen. Mit positiven Überwachungskosten resultiert aus einem Risikoschock, der sich auf die Wohnimmobilienproduktion auswirkt, ein moderater Rückgang der BIP und der Arbeit. Der Konsum reagiert positiv, was mit den Modellen, die einen investitionsspezifischen Technologieschock enthalten (z.B. Greenwood, Hercowitz und Krusell (2000)) konsistent ist. Abbildung 5 gibt die Resonanzfunktionen für den Hauspreis, den Landpreis und die Investitionen in Wohnimmobilien auf die beiden Schocks wieder. Ein Technologieschock auf den Bausektor resuliert in niedrigeren Kosten für Wohnimmobilieninputs, sodass der Preis für Wohnimmobilien sinkt. Der Preis für Land, welcher ein fester Faktor ist, steigt hingegen. Folglich steigt der Gesamtwert eines Hauses auf einem Stück Land, wenn

\footnotetext{
${ }^{23}$ Man kann die Überwachungskosten von 0,25 als den Verlust von 25 Prozent der gesamten Wohnimmobilienproduktion auf Grund von Bankrotten interpretieren.

${ }^{24}$ Die Skalierung für diese Impuls-Antwort-Funktionen ist in Prozent.
} 
ein positiver Technologieschock im Konstruktionssektor auftritt. Bei einem Unsicherheitsschock verursacht der daraus resultierende Rückgang im Angebot von Wohnimmobilien einen Rückgang der Nachfrage nach dem festen Faktor (Land) und einen Anstieg des Preises vom Endprodukt (Wohnimmobilie). Die Antwort der Investitionen in Wohnimmobilien sowohl auf einen Technologieschock als auch auf einen Risikoschock ist genau so, wie man sie erwartet: ein Schock mit Anstieg der Unsicherheit (Risiko) erzeugt einen scharfen Rückgang der Investitionen in Wohnimmobilien, da dies ein wesentlicher Input zur Endproduktion von Häusern ist.

\section{Abbildung 5 hier einfügen}

Zum Abschluss dieses Abschnitts ist ein Wort der Vorsicht zur Interpretation der quantitativen Beträge nötig, die bei den Reaktionen zu sehen sind. Insbesondere ist zu beachten, dass die Reaktion von Hauspreisen auf einen Anstieg der Produktivität im Konstruktionssektor größer ist als die Reaktion auf einen Risikoschock. Man könnte schließen, dass der Wohnimmobiliensektor und Risikoschocks eine untergeordnete Rolle in der Bewegung der Hauspreise spielen. Jedoch wäre eine solche Schlussfolgerung nicht korrekt, falls man die Ergebnisse des vollen Modells (d.h. wenn die gesamte sektorale Technologie und die Risikoschocks vorhanden sind) betrachtet: Die Ergebnisse in Tabelle 1 weisen darauf hin, dass der Risikoschock der treibende Mechanismus ist, der dazu führt, dass sowohl die Volatilität der Hauspreise als auch der Investitionen in Wohnimmobilien angepasst werden.

\section{Einige abschließende Bemerkungen}

Aktien- oder Immobilienblasen zeitnah zu erkennen ist extrem schwierig. Dem Zusammenbruch des U.S. Subprime Hypothekenmarkts im Jahr 2007 wird oft die Schuld an der letzten Immobilienkrise in verschiedenen Industrieländern auf der ganzen Welt gegeben. Die Medien und viele Akademiker behaupten, dass die Zyklen des jüngsten weltweiten Wohnimmobilienbooms und der Wohnimmobilienkrise ein klassisches Beispiel für eine Immobilien-"Blase" waren. In diesem 
kurzen Artikel stellen wir einige der jüngsten empirischen Arbeiten vor, die zeigen, dass diese großen Schwankungen in den Hauspreisen aber weltweit weitgehend durch ökonomische Grundsätze verursacht wurden.

Überdies stellen wir einen anderen Bereich der Literatur vor, welcher sich auf die Rolle der Unsicherheit bei den Immobilien- und Konjunkturzyklen konzentriert. DLS (2011) zeigen zuerst, dass Risikoschocks auf den Wohnimmobilien produzierenden Sektor eine quantitativ große Rolle für die Unsicherheit über die Immobilien- und Konjunkturzyklen implizieren. Als zweites kann ihr Modell die meisten der typischen Merkmale der stilisierten Fakten von Wohnimmobilien erklären, insbesondere, dass Hauspreise und Investitionen in Wohnimmobilien volatiler als der Output sind. Folglich schließen DLS (2011), dass die Rolle der Unsicherheit auf den Immobilienmärkten wichtige quantitative Prognosen hat und somit ein wichtiger Impulsmechanismus in der Literatur zum Kreditkanal sein könnte, welcher bisher übersehen wurde.

Zusammenfassend zeigen wir, dass die Hauptmerkmale der Charakteristika von Wohnimmobilien durch grundlegende ökonomische Schocks eher erklärt werden können als durch Irrationalität der Konsumenten und Investoren. Falls man Widerspruch dagegen erhebt, dass die jüngsten dramatischen Bewegungen der Wohnimmobilienpreise auf ökonomischen Grundlagen basieren, dann haben wahrscheinlich alle Strategien mit dem Ziel, die Hauspreise durch z.B. Interventionen auf dem Kreditmarkt zu beeinflussen nur einen sehr kleinen Einfluss. Falls andererseits die Strategien des Staats darauf abzielen, die Unsicherheit zu verringern und somit die Volatilität der Wohnimmobilienpreise zu mindern, dann könnten, wie wir in DLS (2011) gesehen haben, die Interventionen des Staats in diversen Formen eine wichtige Rolle bei der Vermeidung von zukünftigen Finanz- und Immobilienkrisen spielen. 


\section{Data appendix}

- Gross Domestic Product (GDP), Personal Consumption Expenditures (PCE), Aggregate of gross private domestic investment (Non-RESI), Residential gross private domestic investment (RESI), and the Price Indexes for private residential Investment (PRESI) are all from the National Income and Product Accounts Tables (NIPA) at the Bureau of Economic Analysis.

- House Price Index. (HPI): Constructed based on conventional conforming mortgage transactions obtained from the Federal Home Loan Mortgage Corporation(Freddie Mac) and the Federal National Mortgage Association (Fannie Mae). Source: The Office of Federal Housing Enterprise Oversight (OFHEO).

- The Bank for International Settlements for the selected European countries. 


\section{References}

André, C. (2010), "A Bird's Eye View of OECD Housing Markets", OECD Economics Department Working Papers, No. 746, OECD Publishing. doi: 10.1787/5kmlh5qvz1s4-en

Aoki, Kosuke, J. Proudman, and J. Vlieghe, (2004), "House Prices, Consumption, and Monetary Policy: A Financial Accelerator Approach." Journal of Financial Intermediation, Vol. 13, No. 4, pp. 414-35.

Baker, Dean (2006) "The Menace of an Unchecked Housing Bubble," The Economists' Voice: Vol. 3 : Iss. 4, Article 1. Available at: http://www.bepress.com/ev/vol3/iss4/art1

Carlstrom, C.T. and T.S. Fuerst, (1997), "Agency Costs, Net Worth, and Business Fluctuations: A Computable General Equilibrium Analysis," American Economic Review, Vol. 87, pp. $893-910$.

Carlstrom, C.T. and T.S. Fuerst, (1998), "Agency Costs and Business Cycles", Economic Theory, Vol. 12, pp. 583-597.

Christiano, L, M. Eichenbaum, and C.L. Evans, (2005), "Nominal Rigidities and the Dynamic Effects of a Shock to Monetary Policy," Journal of Political Economy, Vol. 113, No.1, pp. 1-45.

Christiano, L., R. Motto, and M. Rostagno, (2008), "Financial Factors in Business Cycles", working paper.

Davis, Morris and J. Heathcote, (2005), "Housing and the Business Cycle", International Economic Review, Vol. 46, No. 3, pp. 751 - 784.

Dorofeenko, Victor, Gabriel Lee and Kevin Salyer, (2008), "Time Varying Uncertainty and the Credit Channel", Bulletin of Economic Research, Vol. 60, No. 4, pp. 375-403

- (2011), "Risk Shocks and Housing Markets," manuscript.

Dudley, William (2010), "Asset Bubbles and the Implications for Central Bank Policy", http://www.newyorkfed.org/newsevents/speeches/2010/dud100407.html

Favilukis, Jack, Sydney Ludvigson and Stijn Van Nieuwerburgh, (2010), "The Macroeconomic Effects of Housing Wealth, Housing Finance, and Limited Risk-Sharing in General Equilibrium", NYU Department of Economics, manuscript.

Garriga, C., (2010), "The Role of Construction in the Housing Boom and Bust in Spain", Federal Reserve Bank of St. Louis. manuscript.

Glaeser, Edward, Joseph Gyourko, and Albert Saiz, (2008), "Housing supply and housing bubbles", Journal of Urban Economics Vol. 64, Issue 2, pp. 198-217

Glaeser, Edward, Joshua Gottlieb , and Joseph Gyourko, (2010), "Did Credit Market Policies Cause the Housing Bubble?" Rappaport Institute/Taubman Center Policy Briefs, September Issue.

Greenwood, J., Hercowitz, Z. and Krusell, P.,(2000). "The Role of Investment Specific Technological Change in the Business Cycle", European Economic Review, Vol. 44, pp. 91-115.

Himmelberg, Charles, Christopher Mayer and Todd Sinai (2005), "Assessing High House Prices: Bubbles, Fundamentals, and Misperceptions," Journal of Economic Perspectives, Vol. 19, No. 4, pp 67 - 92. 
Hubbard, Glenn and Christopher Mayer, (2009), "The Mortgage Market Meltdown and House Prices", The B.E. Journal of Economic Analzsis \& Policy, Vol. 9, Iss. 3, article 8, pp $1-45$.

Iacoviello, Matteo, "House Prices, Borrowing Constraints and Monetary Policy in the Business Cycle" (2005), American Economic Review, Vol. 95, No. 3, pp. 739-764.

Iacoviello,Matteo and Raoul Minetti,(2008), "The Credit Channel of Monetary Policy: Evidence from the Housing Market" (forthcoming), Journal of Macroeconomics.

Iacoviello, Matteo and S.Neri, (2010), "Housing Market Spillovers: Evidence from an Estimated DSGE model", American Economic Journal: Macroeconomics, Vol. 2, Iss. 2, pp.12564 .

Kahn, James, (2009), "What Drives Housing Prices?" Federal Reserve Bank of New York Staff Reports, No. 345 .

Piazzesi, Monika and Martin Schneider, (2009),. "Momentum Traders in the Housing Market: Survey Evidence and a Search Model," American Economic Review, Vol. 99. No.2, pp. 40611.

Poterba, James, (1984), "Tax Subsidies to Owner-occupied Housing", Quarterly Journal of Economics, Vol. 99. No. 4, pp. 729 - 752.

Shiller, Robert J. (1989), Market Volatility, Cambridge, MA: The MIT Press.

- (2009),"Unlearned Lessons from the Housing Bubble," The Economists' Voice: Vol. 6 : Iss. 7, Article 6.

- (2009), "Why We'll Always Have More Money Than Sense" in Newsweek

Sommer, Kamila, Paul Sullivan and Randal Verbrugge, (2010), "Run-up in the HOuse Price-Rent Ratio: How much Can be Explained by Fundamentals?", manuscript.

Stiglitz, Joseph E, (1990), "Symposium on Bubbles", Journal of Economic Perspectives, Vol. 4. No. 2, pp. 13 - 18. 